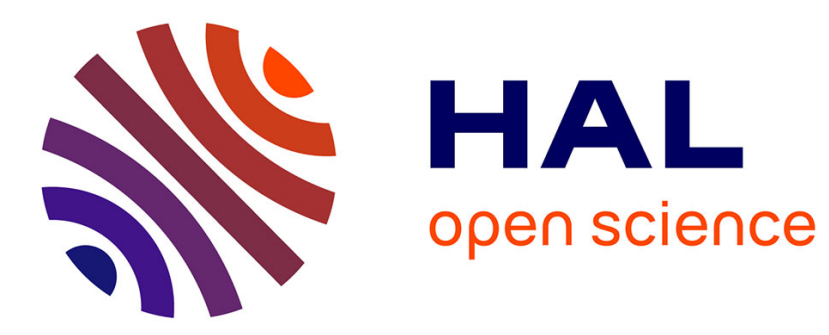

\title{
Wake-up radio architecture for home wireless networks
}

Florin Hutu, Aissa Khoumeri, Guillaume Villemaud, Jean-Marie Gorce

\section{To cite this version:}

Florin Hutu, Aissa Khoumeri, Guillaume Villemaud, Jean-Marie Gorce. Wake-up radio architecture for home wireless networks. IEEE Radio Wireless Symposium, Jan 2014, Newport Beach, United States. hal-00920899

\section{HAL Id: hal-00920899 \\ https://hal.inria.fr/hal-00920899}

Submitted on 19 Dec 2013

HAL is a multi-disciplinary open access archive for the deposit and dissemination of scientific research documents, whether they are published or not. The documents may come from teaching and research institutions in France or abroad, or from public or private research centers.
L'archive ouverte pluridisciplinaire HAL, est destinée au dépôt et à la diffusion de documents scientifiques de niveau recherche, publiés ou non, émanant des établissements d'enseignement et de recherche français ou étrangers, des laboratoires publics ou privés. 


\title{
Wake-up radio architecture for home wireless networks
}

\author{
Florin Hutu, Aissa Khoumeri, Guillaume Villemaud, Jean-Marie Gorce \\ florin-doru.hutu@insa-lyon.fr
}

Université de Lyon, INRIA, INSA-Lyon, CITI-INRIA, 6, avenue des Arts, F-69621, Villeurbanne

\begin{abstract}
In this paper it is presented and validated by simulation a wake-up radio receiver (WuRx), allowing to both addressing and wake-up a main radio, with best performance but more energy consuming. The main goal is to reduce the total energy consumption of a home multimedia network by switching off the data module of each equipment during the periods when it is not used. The wake-up receiver is designed to be able to detect a wake-up signal which has a frequency signature and so, it is avoided to activate all data interfaces but only the one of interest.
\end{abstract}

Index Terms-wake-up radio, wireless sensor networks, energy consumption

\section{INTRODUCTION}

More and more home multimedia networks are formed by equipment having a low usage rate but being always switched on. This implies important electrical energy consumption and it is estimated that a simple extinction of the data interface leads to about $40 \%$ of energy reduction. More generally, idle mode listening, typically used in wireless sensor networks (WSN), will impact the node energy consumption. This can be particularly suboptimal when the data traffic is very sporadic.

Efforts to reduce energy consumption are undertaken at different levels like medium access protocol (MAC) by dynamically adapting the listen/sleep duty cycle. At physical layer, efforts are made in order to propose energy efficient waveforms and codes or optimized radio-frequency (RF) front-ends. In [1], instead of a stack-up front-end approach, a unique front-end is used to simultaneously treat two frequency bands and so reduce the energy consumption by reducing the number of components. Another approach that is taken into account in order to increase the energy efficiency is the cross layer optimization, by exploiting the interaction between different layers, from physical to application layer [2].

All these efforts converge to the conclusion that, at the physical layer, control mechanisms must be created in order to activate or deactivate a part of or the entire radio front-end. In wireless sensor networks in general and in home multimedia networks in particular, in order to control the state of the radio front-end, a second front-end, with lower energy consumption compared to the main one is used. The overlay network, composed by the low energy front-ends, will only convey the information on which set of nodes must communicate at a certain time.
The aim of this paper is to propose a wake-up radio architecture activating the main radio only in the presence of an identifier which is a frequency fingerprint and consequently, no base-band treatment is needed. This implies reduced latency time and energy consumption in stand-by mode while eliminating the need for a special TX to emit the identifier.

The novelty of the proposed architecture is that the decision is taken at radio-frequency circuit level and so, the expected DC power consumption is less than that announced by the other circuits presented in the literature, for the same characteristics in terms of sensitivity and robustness.

\section{State of The ART ON WAKE-UP RADio ARCHITECTURES}

Radio-frequency wake-up systems can be divided in two categories. The first group is represented by rectenna systems first presented in [3]. This kind of wake up systems detect only a certain power level on a radio channel. Despite its very low power consumption, the main drawback is that it cannot make the difference between a noisy channel and a real wake-up signal and consequently, its sensibility is very degraded. The wake up signal is transformed into a DC one by using charge pumps or voltage multipliers. In [3], a diode based architecture is presented and in [4], MOS transistors replace the diodes.

Another group may be formed by classical radiofrequency front-ends and mainly, the energy consumption will be correlated to the kind of chosen modulation. Consequently there are two types of radio architectures. The first one is the classical homodyne architecture that transposes the received signal in the base-band [5]. The second type is the on-off keying (OOK) modulation dedicated architecture which receiver is an envelope detector who recovers the digital signal as the amplitude of the radio frequency one. This last architecture is proposed in most of wake-up radio applications because of its low complexity and low energy consumption.

Table I gathers some of the noticeable wake-up architectures based on OOK modulation. Three comparison parameters are highlighted: firstly the working frequency; most of the WuRx systems are designed for the ISM bands. Then, the DC consumed power and the sensitivity (defined 
as the minimum identification signal discernable by the WuRx) are also compared.

\begin{tabular}{|c|c|c|c|}
\hline Publication & Frequency & DC Power & Sensitivity \\
\hline \hline$[3]$ & $433 \mathrm{MHz}$ & $1.07 \mu W$ & $-51 \mathrm{dBm}$ \\
\hline$[6]$ & $868 \mathrm{MHz}$ & $24 \mu W$ & $-57,1 \mathrm{dBm}$ \\
\hline$[7]$ & $2.4 \mathrm{GHz}$ & $2.59 \mathrm{~mW}$ & $-75 \mathrm{dBm}$ \\
\hline$[8]$ & $433 \mathrm{MHz}$ & $8 \mathrm{nW}$ & $-51 \mathrm{dBm}$ \\
\hline$[9]$ & $868 \mathrm{MHz}$ & $2.7 \mu W$ & $-52 \mathrm{dBm}$ \\
\hline
\end{tabular}

TABLE I

NON-EXHAUSTIVE STATE OF THE ART OF WURX

\section{DESCRIPTION OF THE WAKE-UP ARCHITECTURE DESIGN}

Aiming to develop an ultra-low power wake-up radio for a WLAN-type application, as seen in figure 1, the proposed architecture consists mainly in a wake-up radio receiver associated to the existing 802.11 equipment and monitoring the channel for the wake-up signal. It is supposed that the main radio interface of the receiver $j$ is off and the equipment $i$ initiate a data communication with this equipment. In this case, the $i^{\text {th }}$ data emitter sends the address @ $j$ of the $j^{\text {th }}$ equipment as a frequency pattern. The wake-up radio of the $j^{\text {th }}$ switches on the power supply of the main data front-end and the communication begins while the rest of the equipments remain asleep.

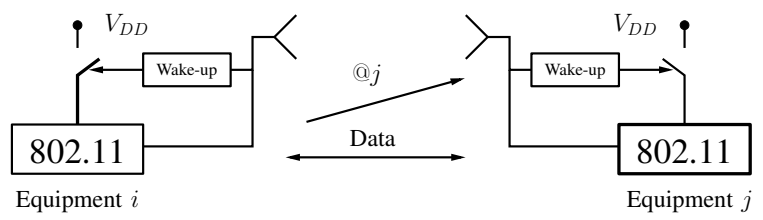

Fig. 1. Wake-up mechanism

Most of the home multimedia equipment has an 802.11 OFDM-compliant transceiver; it is considered that the identifier is emitted by this classical emitter, using a particular and predetermined sub-carrier pattern as the ID. Only a set of $n$ groups of adjacent sub-carriers are used in the total channel bandwidth, other sub-carriers being forced to zero. Figure 2 presents an identifier formed on a $20 \mathrm{MHz}$ channel around $2.45 \mathrm{GHz}$ and grouping the sub-carriers in $n=4$ equally spaced subsets.

The 802.11n standard imposes the OFDM modulation on 64 sub-carriers, with $312.5 \mathrm{kHz}$ spacing between them. The proposed wake-up radio allows to identify the equipment to wake-up by using two multi-band filters having complementary frequency patterns, as can be seen in figure 3. The filter on the direct path has the same frequency pattern as the identifier.

Two envelope detectors transform the RF signal at the filters output into $V_{D C 1}$ and $V_{D C 2}$, two DC voltages. If the

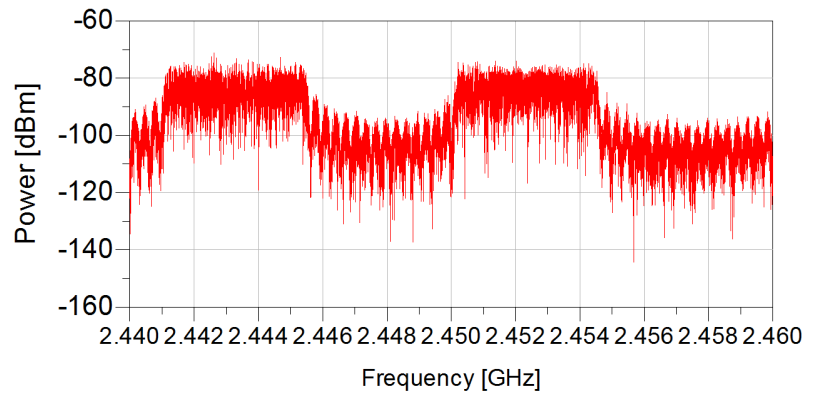

Fig. 2. Example of identifier

received signal is the identifier, then the difference between the two DC voltages is greater than $V_{\text {threshold }}$ voltage and then the $V_{C O M}$ output voltage is at the highest level. This last voltage allows switching on the main front-end supply and the data transfer may begin.

One of the key points of the proposed architecture is the global sensitivity given by the envelope detector sensitivity. Generally, a zero polarization Schottky diode is used and in this case, the sensitivity is defined by the lowest detected $\mathrm{RF}$ power level which gives $8 \mathrm{~dB}$ output signal-to-noise ratio [10]. The state of the art gives an expected sensitivity of $-50 \mathrm{dBm}$. Another important parameter of the Schottky diode is their responsivity defined as the DC voltage at the output of the envelope detector function of the input RF power level.

Range estimation can be calculated. To do so, it is considered that the emitting antenna has enough gain to compensate the insertion losses of the multi-band filters and the power dividers used to convey the received signal to the filters. The minimum received signal is approximated by the Schottky diodes sensitivity. Because of the considered application, the first approach is to model the propagation channel as an indoor channel having a path loss exponent between 1.6 and 3 and a shadowing margin equal to $5 \mathrm{~dB}$. It is also considered that the identifier is emitted with a maximum equivalent isotropically radiated power (EIRP) of $20 \mathrm{dBm}$ and the receiving antenna is omnidirectional with $2 \mathrm{dBi}$ gain. Under these conditions,

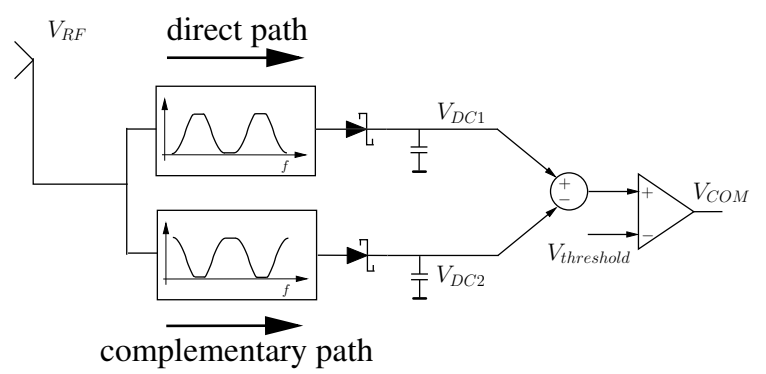

Fig. 3. Block diagram of the wake-up receiver 
the estimated range is between 8 and 48 meters.

\section{Simulation RESUlts}

In order to validate the proposed wake-up receiver architecture, circuit-system co-simulations using ADS Agilent's software where performed. The multi-band filters were considered having $1 \mathrm{~dB}$ insertion loss and an out of band attenuation of $-50 \mathrm{~dB}$. As power detectors, zero polarization Schottky diode HSMS2850 of Agilent have been put in series with $10 \mathrm{nF}$ capacitors.

Simulations were also performed in order to establish the responsivity of the Schottky diode for an input signal at $2.45 \mathrm{GHz}$. Beforehand, a matching circuit was calculated and simulated for a RF power level equal to the diode sensitivity. The DC output voltage varies from $550 \mu \mathrm{V}$ to $420 \mathrm{mV}$ which is considered to be sufficient to drive the substractor's inputs. In order to obtain the $V_{\text {com }}$ output, a trigger was used. The substractor and the Schmidt trigger are simulated using op-amp models and the trigger's threshold was set the manner that the output $V_{\text {com }}$ switches for an $-50 \mathrm{dBm}$ identifier power level.

Figure 4 presents the output of the envelope detector for the direct and complementary paths in presence of the identifier presented in figure 2. The power level is set to $-40 \mathrm{dBm}$ and the identifier is sent during $400 \mathrm{nsec}$. As it can be seen, when the capacitor is charged, a quasicontinuous voltage is obtained. As expected, the received power level at the complementary path is weaker an so, the DC voltage is less than in direct path.

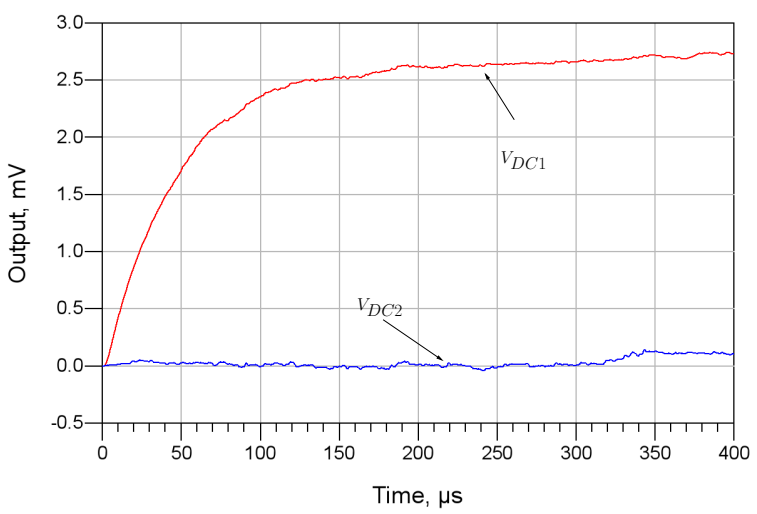

Fig. 4. Power detector's outputs on direct and complementary paths

The two outputs in figure 4 are substracted and amplified. The result allows to switch the $V_{\text {com }}$ trigger output to a positive voltage, as seen in figure 5 .

\section{CONCLUSion}

In this paper, a wake-up radio architecture has been presented and validated by simulations at circuit level. The identification of the equipment to wake-up is done by detecting the RF power level at the output of two multi-band

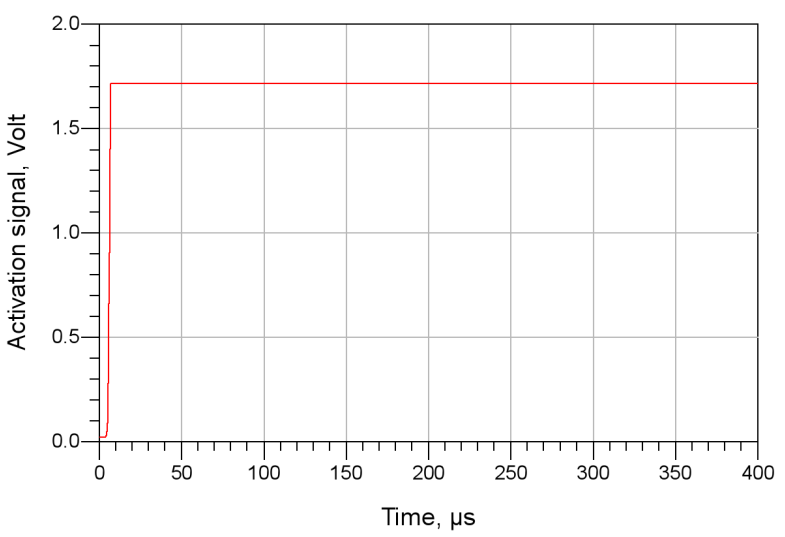

Fig. 5. Activation signal: $V_{C O M}$ voltage

filters, having a complementary bandpass. Complementary studies are undertaken in order to quantify the robustness of the proposed architecture with respect to false wakeups. Moreover, a prototype will be manufactured in order to have measurements on range, delay and energy consumption.

\section{REFERENCES}

[1] I. Burciu, G. Villemaud, J. Verdier, and M. Gautier, "Low power front-end architecture dedicated to the multistandard simultaneous reception," International Journal of Microwave and Wireless Technologies, vol. 2, no. 6, pp. 505 - 514, 2011.

[2] G. Miao, N. Himayat, Y. G. Li, and A. Swami, "Cross-layer optimization for energy-efficient wireless communications: a survey," Wireless Communications and Mobile Computing, vol. 9, no. 4, pp. 529 - 542, April 2009.

[3] L. Gu and J. A. Stancovic, "Radio-triggered wake-up for wireless sensor networks," Real-Time Systems, vol. 29, pp. 157 - 182, 2005.

[4] H. Kim, H. Cho, Y. Xi, M. Kim, S. Kwon, J. Lim, and Y. Yang, "CMOS passive wake-up circuit for sensor network applications," Microwave and Optical Technology Letters, vol. 52, no. 3, pp. 597-600, 2010. [Online]. Available: http://dx.doi.org/10.1002/mop.24982

[5] N. M. Pletcher, S. Gambini, and J. Rabaey, "A $52 \mu W$ wake-up receiver with $-72 \mathrm{dBm}$ sensitivity using an uncertain-IF architecture," IEEE Journal of Solid-State Circuits Circuits, vol. 44, no. 1, pp. 269-280, January 2009

[6] C. Hambeck, S. Mahlknecht, and T. Herndl, "A $2.4 \mu W$ wakeup receiver for wireless sensor nodes with $-71 \mathrm{dBm}$ sensitivity," in IEEE International Symposium on Circuits and Systems, 2011.

[7] M. Vidojkovic, X. Huang, P. Harpe, S. Rampu, C. Zhou, L. Huang, K. Imamura, B. Busze, F. Bouwens, M. Konijnenburg, J. Santana, A. Breeschoten, J. Huisken, G. Dolmans, and H. de Groot, "A 2.4GHz ULP OOK single-chip transceiver for healthcare applications," in 2011 IEEE International Solid-State Circuits Conference Digest of Technical Papers (ISSCC), feb. 2011, pp. $458-460$.

[8] S. J. Marinkovic and E. M. Popovici, "Nano-power wireless wakeup receiver with serial peripheral interface," IEEE Journal on selected areas in communications, vol. 29, no. 8, pp. 1641 - 1647, September 2011.

[9] G. U. Gamm, M. Sippel, M. Kostic, and L. M. Reindl, "Low power wake-up receiver for wireless sensor nodes," in International Conference on Intelligent Sensors, Sensor Networks and Information Processing, 2010.

[10] X. Fan, X. Pei, and X. Xiong, "Zero bias Schottky diodes use in high performance detection circuits," in Electronics and Optoelectronics (ICEOE), 2011 International Conference on, vol. 3, 2011. 